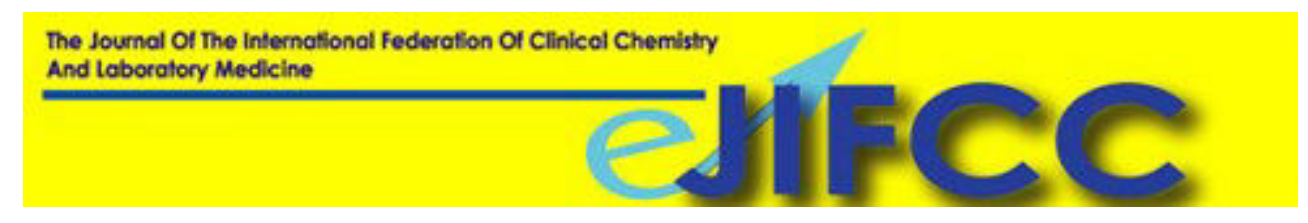

\title{
APOPTOSIS AND AUTOIMMUNITY
}

Assist. Prof. Borut Božič, Ph.D.; Prof. Blaž Rozman, M.D.

University of Ljubljana, Faculty of Pharmacy, Chair for Clinical Biochemistry, Ljubljana, Slovenia

University Medical Centre, Department of Rheumatology, Immunology Laboratory

\subsection{Introduction}

Apoptosis and autoimmunity are two phenomena, which share essential similarities in destroying cells. However, apoptosis is a normal process, while autoimmunity is not. Apoptosis could, at least in some conditions, cause autoimmunity. The failure to appropriately achieve programmed cell death and to clear apoptotic cell fragments is discussed as a key pathogenetic factor leading to autoimmunity.

\subsection{Cell death}

The term "apoptosis" (from Greek apo = from and ptosis = falling) was first mentioned in 1972 in the article of John Kerr and co-workers (Br J Cancer 1972;26:239-57) to distinguish between developmental cell death and necrosis (from Greek nekros $=$ dead) as unprogrammed death of cells due to injury, infection, cancer, infarction or inflammation. Apoptosis is almost invisible in tissue. In contrast, fibrinoid necrosis, which is caused by immune-mediated vascular damage, is marked by deposition of fibrin-like proteinaceous material in arterial walls seen by microscope.

Apoptosis is a process characterized by the plasma membrane blebbing, cytoplasmic and organelle concentration and shrinkage, nuclear chromatin condensation and DNA cleavage at certain internucleosomal sites, producing characteristic 180-200 base pairs fragments. Finally, generated cellular fragments or apoptotic bodies become rapidly ingested by macrophages or neighboring cells. Although cells undergoing apoptosis show distinct morphological characteristics, their rapid clearance generally results in a very small number of cells that can be categorized as apoptotic at any given time. The highly efficient elimination of apoptotic cells occurs fast and without tissue damage or inflammation. Apoptosis is known as programmed cell death, but the linkage between apoptosis and programmed cell death, evolutionarily conserved process was made in early nineties, more than hundred years after Flemming's description of the naturally occurring form of cell death, then called "chromatolysis" (Archiv für Anatomie und Physiologie 1885;221-4). Nowadays two forms of physiological or programmed cell death are distinguished: apoptotic or type I and autophagic or type II. Autophagic cell death includes degradation of the Golgi apparatus, polyribosomes, and endoplasmic reticulum, which precede nuclear destruction. Intermediate filaments and microfilaments are largely preserved; presumably the cytoskeleton is required for autophagocytosis. Vacuolization is a common feature. 


\subsection{Basic molecular mechanisms of apoptosis}

The "decision" to activate a suicidal process is made on the basis of intrinsic or extrinsic apoptotic messages. Intrinsic inducers come from mitochondria or nucleus. Extrinsic inducers are ligands (cytokines) for death receptors on the cell surface. But apoptosis can be induced also by lack of signal molecules produced by other cells, which normally work in combinations to regulate the behavior of the cell. An individual cell requires multiple signals to survive; otherwise it will undergo a programmed cell death.

Irrespective of the initial signal, the core role belongs to a family of proteases that have cysteine at their active site and cleave target proteins at a specific aspartic site. They are called caspases. Since activation of caspases and their cleavage are irreversible, the entrance into apoptosis is in an all-or-none mode, without a possibility to turn back.

Caspases are synthesized in inactive form as procaspases. Each caspase consists of a structurally related molecule with prodomain, a large subunit and a small subunit. The latter two combine to form tetramers after cleavage. When activated, they cleave the aspartic moiety of another procaspases, make them active. Currently 13 different caspases have been described, 11 of them were found in humans. Based on their function and substrate specificity they can be classified into three groups: a/ upstream enzymes, which amplify death signal; b/ CED-3-like caspases (CED = cell death, product of gene ced involved in apoptosis found in Caenorhabditis elegans), which rapidly cleave structural and vital components, and c/ ICE-like caspases (ICE = interleukine-1 beta-converting enzyme), which may be involved in inflammation rather than death.

The leader position among upstream enzymes belongs to initiator procaspases-8. When they are pushed together by adapter proteins, they cleave themselves to active initiator caspases-8. In the enzymatic cascade, caspases' activity is amplified until key reactions are reached:

- Cleavage of a cytoskeleton, making cell circular and less rigid

- Cleavage of nuclear pore proteins, making nuclear envelope more permeable

- Cleavage of nuclear lamins, causing irreversible breakdown of nuclear lamina

- Cleavage of a protein that normally holds a DNase in inactive form

- Cleavage of poly-(ADP-ribose)-polymerase

- Degradation of chromatin and its packaging proteins

\subsection{Regulation of the cell death program}

Apoptosis is not a result of the disbalance between pro- and anti- apoptotic stimuli. The most important regulatory mechanisms in mammalians are: death receptors, caspases, mitochondria, the Bcl-2 family of proto-oncogenes and tumor-suppressor gene p53.

The main death receptors (DR) are CD95 (Fas), CD120a (TNF-RI), DR3, DR4, DR5 and DR6, which are responsive to cytokines belonging to the tumor necrosis factor family (TNF-alfa, lymphotoxin, FasL, ApoL3). The binding of a ligand provokes trimerization of the receptor and recruitment of intracellular adaptor proteins that aggregate initiator procaspase-8. In 
contrast to FasL, TNF-alfa mediated signaling may stimulate cell proliferation through the nuclear factor kappa beta pathway, depending on the cell type, the receptor (CD120a or CD120b) and other regulators. The link between apoptosis and TNF activity shows why abnormal production of TNF plays an important role in several autoimmune diseases (e.g. rheumatoid arthritis, diabetes mellitus, multiple sclerosis).

Mitochondria have a fundamental position in executing apoptosis induced by intracellular signals. When cells are stressed due to physical stimulus (UV or gama radiation), chemical stimulus (genotoxic or cytotoxic drugs, metabolic poisons), or an altered conditions (detachment of cells from the extracellular matrix, cytokine- and growth factordeprivation) mitochondria release pro-apoptotic proteins, including cytochrome c. It binds to an adapter protein Apaf-1, thus activating procaspase- 9 and enzymatic cascade. Additional mitochondrial activities leading to apoptosis are the disruption of electron transport and modification of cellular reduction-oxidation potential.

The most important proto-oncogenes involved in maintaining cell survival and proliferation are those belonging to the $\mathrm{Bcl}-2$ family. Its gene products have different functions: Bcl-2 by itself and Bcl-XL are inhibitors of apoptosis, while Bad, Bax, Bak, and $\mathrm{Bid}$ are promoters. Protein Bad binds and inactivate inhibitors of apoptosis Bcl-2 and Bcl$\mathrm{XL}$. Bid is activator of Bax and Bak, which are initiators of apoptosis through stimulating cytochrome $\mathrm{c}$ release from mitochondria.

Among tumor-suppressor genes, $\mathrm{p} 53$ is the most important. In response to DNA damage, the product p53 can induce apoptosis through the activation of gene encoding cytochrome c releasing proteins.

Inhibitors of apoptosis family (IAP) inhibit apoptosis by deterrence of activity of some caspases and by prevention of activation of some procaspases.

Some other modifiers of apoptosis have been reported. Nitric oxide may increase the mitochondrial membrane potential and may chemically modify cytochrome c, both leading to promote the release of cytochrome c. On the other hand, nitric oxide may decrease the activity of caspases by induction of their S-nitrosylation. High levels of reactive oxygen species promote lipid peroxidation, macromolecular damage and necrotic cell death, while lower levels of reactive oxygen species promote changes in cell signaling that result in apoptosis.

\subsection{Apoptosis as causal factor for autoimmunity}

Apoptosis is a process "where too much or too little can lead to autoimmunity" (Grodzitsky T, Elkon KB. Mt Sinai J Med 2002;69:208-19). Indeed, the capacity for unrestricted self-renewal is a dangerous property for any cell to possess, and many cases of leukemia occur through mutations that confer this capacity. On the other hand, too much apoptosis of beta isle cells of the pancreas is implicated in the pathogenesis of diabetes mellitus.

Activation of naive T cells by antigen results in the massive expansion of antigen specific $T$ cells. The majority of $T$ cells die due to apoptosis, while a small number survive and 
differentiate into memory cells. This cell destiny is crucial for our understanding of how autoimmunity is evaded. Some autoreactive cells may escape natural apoptosis and represent continuous threat with potential autoimmune response. But also apoptotic cells do not disappear after phagocytosis. Ingredients of the apoptotic blebs indeed survive the intracellular processing and are recycled to the membrane of the phagocyte. Massive apoptosis overloading the phagocytic capacity may trigger an autoimmune reaction through presentation of nucleosomes to the immune system. Even after death cell components keep being perceptible to the immune system. Natural antibodies with activity to apoptotic blebs, or the first waves of autoantibodies, which bind with relative low affinity cell debris (noticeable also after infection) may increase depletion efficiency via opsonisation; but extensive overload with apoptotic corps, extensive presentation of nucleosomes to lymphocytes and excessive amount of autoreactive antibodies could disbalance the immune/apoptotic homeostasis and contribute to the pathogenesis of systemic and organ specific autoimmune disease. Apoptosis has traditionally been thought of as a non-inflammatory process, which does not induce an immune response. However, recent studies indicate that apoptotic cells can be involved in (auto) immune processes. They can display autoreactive antigen in their surface blebs, they can activate dendritic cells and they can induce the formation of autoantibodies.

\subsection{Apoptosis and autoimmune diseases}

In systemic autoimmune diseases, especially in systemic lupus erythematosus, autoantibodies against a palette of intra-cellular antigens are found. It has been suggested that humoral response may be driven by the products of apoptotic cells with the central importance of the nucleosomes as potential (auto)antigens. It was shown that after apoptosis, induced in keratinocytes following UV light exposure, the cells express on their surface (in blebs) several intracellular antigens such as Ro, La, ribosomes and nucleosomes; but an increased number of nuclear antigens per se is not sufficient to induce lupus-like autoimmune response. Autoreactive B and T cells, escaped from natural apoptosis might represent additional necessary condition. Caspase activity in apoptotic cells may lead to presentation of cryptic epitopes or neoepitopes to which the immune system is not tolerant: modified antigens (phosphorilated, citrulinated, acetylated) may lead to an increased immunogenicity. Direct access to the cytosol of antigens derived from the processing of engulfed apoptotic cell corpses has been reported in activated phagocytes.

This event results in proteosome-dependent processing of apoptotic cell-associated antigens, translocation into the endoplasmatic reticulum and loading into MHC class I molecules. Antigens, contained in theengulfed apoptotic remnants may use both MHC class I and class II molecule pathways as well as other less-conventional antigen presentation pathway. Observed decreased NF-KB (p65) expression in lupus patients may be linked with inappropriate proteasome-dependent processing, promoting autoimmune response, especially when dendritic cells are involved in phagocytosis of apoptotic cells. Communication among uncleared apoptotic cells and interferon- $\alpha$ excreted dendritic cells plays an important role in the pathogenesis of disease. Increased CD95, CD95L, and CD40L expression, decreased TGF $\beta$ secretion, defects in phagocytosis are fragments of our knowledge, which suggest a strong involvement of the apoptotic processes in the 
pathogenesis of systemic lupus erythematosus.

Antiphospholipid syndrome is characterized by autoantibody-dependent defects in haemostasis, leading to arterial or venous thrombosis. The list of factors, involved in the coagulation process and possible clearance of dying cells, is growing. Among several receptor molecules, which were shown to mediate uptake of apoptotic cells, was also CD68 receptor, which is a receptor for oxidized low density lipoproteins, not yet characterized phosphatidylserine receptor and phosphatidylserine by itself. Activated platelets, which selectively expose phosphatidylserine, are efficiently opsonised by naturally occurring antiphospholipid antibodies. Anti- $\beta 2$-glycoprotein antibodies are one of active opsonins for apoptotic cells, which increase the efficiency of the internalization of apoptotic cell corpses, and preferentially direct them to MHC class II loading compartments. On the other hand, annexin 5 has been demonstrated to interfere with the recognition and clearance of dying cells, increasing their immunogenicity in vivo.

Sjoegren's syndrome is a lymphoproliferative disease characterized by a destructive mononuclear cell infiltration in salivary and lachrymal glands. In animal models of MRL mice, homozygous for lymphoproliferative mutation (Ipr/lpr), salivary gland lymphoid infiltration similar to human Sjoegren's syndrome has been found. In experimental as well as in human Sjoegren's syndrome high levels of CD95/CD95L expression were found in salivary glands, suggesting a mechanism of apoptotic cell death.

Diabetes mellitus type 1 results from an organ-specific autoimmune mediated loss of insulin-secreting $\beta$ cells. This chronic destructive process involves both cellular and humoral components detectable in the peripheral blood, months or even years before the onset of clinical diabetes. The studies from the last ten years suggested that the neonatal wave of $\beta$-cell apoptosis might provide autoantigens necessary for triggering $\beta$ cell directed autoimmunity. In animal models increased TNF $\alpha$ mediated apoptosis has been found, which has been explained by selective nitric oxide-mediated upregulation of functional CD95 molecules on $\beta$ cells that are subsequently killed by CD95L-producing $T$ cells.

Hashimoto's thyroiditis is a chronic autoimmune disease characterized by a progressive destruction of thyroid epithelial cells and reduced production of thyroid hormones. In normal thyroid an exceptionally low rate of apoptosis is found, resulting in slow biological turnover of thyrocytes. CD95 is weakly or not at all expressed in normal thyrocytes. During inflammation interleukin-1 $\beta$ and interferon- $\alpha$ induce massive CD95 upregulation. Simultaneously expressed CD95 receptor and its ligand cause apoptotic cell death by paracrine or autocrine mechanisms, while infiltrating $T$ cells do not seem to play an executive role.

Ulcerative colitis is a chronic inflammatory gut disease with mucosal alterations and the loss of colonic epithelium. The presence of apoptotic corps in the crypt epithelium suggests an involvement of apoptosis. The expressions of CD95 on epithelial cells in ulcerative colitis and normal enterocytes are comparable. It has been proposed that possible increased sensitivity to CD95 provokes apoptosis: epithelial cell death is mediated by CD95L-expressing lamina propria lymphocytes. 
Multiple sclerosis is a progressively demielinating disease of the central nervous system. Destruction of oligodendrocytes has not been completely clarified, but upregulation of CD95/CD95L pathway seems to play a major role, most likely by the initiation of apoptosis at the site of inflammation.

Some examples exist with decreased apoptosis and autoimmunity. In humans decreased CD95 apoptosis has been found in Canale Smith syndrome, including nonmalignant lymphoproliferation with autoimmunity. A defective clearance due to mutations in CD95 proteins results in prolonged survival of potentially autoreactive lymphocytes. High levels of soluble CD95, found in rheumatoid arthritis patients, contribute to inhibition of synoviocyte and inflammatory cell apoptosis. An inadequate apoptosis due to defective CD95 may promote extended survival of synoviocytes. Additionally, their responsiveness to CD95L is decreased by TGF $\beta$, interleukin-1 $\beta$ and TNF- $\alpha$. Both lead to growth of hyperplastic pannus and destruction of cartilage.

\subsection{Conclusion}

Apoptosis or programmed cell death represents a natural way to eliminate unwanted cells. The elimination does not occur without any vestige and reactions. Even more, evidence has been accumulated showing that apoptotic death is involved in (auto)immune response, leading to systemic and organ specific autoimmune diseases. We are still far from the complete understanding and interpretation of all signals and processes in the course of apoptosis and their influences on autoimmunity. Faults at different stages, including defficiencies in the release or in response to endogenous molecules excreted by uncleared dying cells seem to be involved.

\section{Literature}

1. Alberts B, Johnson A, Lewis J, Raff M, Roberts K, Walter P. Molecular biology of the cell. 4th ed., Garland Science, 2002.

2. Kalden JR, Herrmann M. Apoptosis and autoimmunity. From mechanisms to treatments. Wiley-VCH, Weinheim 2003.

3. Horvitz HR. Worms, life and death. Nobel Lecture, Dec 8, 2002.

4. Zakeri Z, Lockshin RA, Benitez-Bribiesca L (eds). Mechanisms of cell death. The New York Academy of Sciences, New York 1999; 887.

5. Grodzitsky T, Elkon KB. Apoptosis: A case where too much or too little can lead to autoimmunity. The Mount Sinai Journal of Medicine 2002; 69:208-19.

6. Rovere-Querini Dumitriu IE. Corpse disposal after apoptosis. Apoptosis 2003; 8:469-79.

7. Hayashi T, Faustman DL. Implications of altered apoptosis in diabetes mellitus and autoimmune disease. Apoptosis 2001; 6:31-45. 